\title{
EXIT PROBLEMS FOR REFLECTED MARKOV-MODULATED BROWNIAN MOTION
}

\author{
LOTHAR BREUER, ${ }^{*}$ University of Kent
}

\begin{abstract}
Let $(\mathcal{X}, \mathcal{g})$ denote a Markov-modulated Brownian motion (MMBM) and denote its supremum process by $\&$. For some $a>0$, let $\sigma(a)$ denote the time when the reflected process $y:=8-\mathcal{X}$ first surpasses the level $a$. Furthermore, let $\sigma_{-}(a)$ denote the last time before $\sigma(a)$ when $\mathcal{X}$ attains its current supremum. In this paper we shall derive the joint distribution of $S_{\sigma(a)}, \sigma_{-}(a)$, and $\sigma(a)$, where the latter two will be given in terms of their Laplace transforms. We also provide some remarks on scale matrices for MMBMs with strictly positive variation parameters. This extends recent results for spectrally negative Lévy processes to MMBMs. Due to well-known fluid embedding and state-dependent killing techniques, the analysis applies to Markov additive processes with phase-type jumps as well. The result is of interest to applications such as the dividend problem in insurance mathematics and the buffer overflow problem in queueing theory. Examples will be given for the former.
\end{abstract}

Keywords: Markov-modulated Brownian motion; reflection; exit problem; Markov additive process

2010 Mathematics Subject Classification: Primary 60J25

Secondary 60G51; 60J55

\section{Introduction}

Markov additive processes are a powerful generalisation of Lévy processes, becoming more and more popular in stochastic modelling. Based on an underlying finite-state Markov process $\mathcal{g}$, called the phase process, the level process $\mathcal{X}$ evolves like a Lévy process for which the parameters change in time according to the phase process $\mathcal{g}$. Furthermore, phase changes (i.e. jumps of $\mathcal{g}$ ) may induce jumps of the level process $\mathcal{X}$. The joint process $(\mathcal{X}, \mathcal{g})$ is called a Markov additive process (MAP).

A textbook introduction to MAPs is given in [2, Chapter XI]. First passage times (or the one-sided exit problem) are derived via martingales in [3] and solved iteratively in [6]. The two-sided exit problem is solved in [17] for MAPs with phase-type jumps (PH-MAPs). The class of PH-MAPs is dense within the class of all MAPs; see [4, Proposition 1]. The GerberShiu function (which is quite popular in insurance mathematics) has been derived in [7] for the same class of MAPs.

It is this class of MAPs for which we wish to solve the following problem. Denote the supremum process of $\mathcal{X}$ by $\&$. For some $a>0$, let $\sigma(a)$ denote the time when the reflected process $\mathcal{Y}:=\delta-\mathcal{X}$ first surpasses the level $a$. Furthermore, let $\sigma_{-}(a)$ denote the last time before $\sigma(a)$ when $\mathcal{X}$ attains its current supremum. We shall derive the joint distribution of $S_{\sigma(a)}, \sigma_{-}(a)$, and $\sigma(a)$, where the latter two will be given in terms of their Laplace transforms.

Received 18 January 2011; revision received 12 January 2012.

* Postal address: Institute of Mathematics and Statistics, University of Kent, Canterbury, CT2 7NF, UK.

Email address: 1.breuer@kent.ac.uk 
This extends recent results for spectrally negative Lévy processes [20] to PH-MAPs. Even for the common subset of Lévy processes with phase-type jumps, the approach in this paper may be advantageous, since the scale functions (which are the main ingredients in the formulae) are given explicitly.

The result is of interest to applications such as the dividend problem in insurance mathematics and the buffer overflow problem in queueing theory. An algorithmic solution for the time to buffer overflow in a Markov additive framework is given in [3, Section 6]; see also [5]. A partial result of the present paper in the context of queueing theory is contained in [8]. An algorithmic solution for the expectation of the total dividend payments before ruin is presented in [11]. Moments of dividend payments in a Markov additive risk model without Brownian component are derived in [18].

The analysis is performed mainly by matrix-analytic methods using probabilistic arguments wherever possible. This naturally results in formulae containing matrices which are to be computed via fixed-point iterations. We shall present examples for the simple cases allowing explicit scalar solutions. This restriction is due to the circumstance that only for these cases are there solutions in the literature which can be compared with the results in the present paper.

It is well known that exit problems for PH-MAPs can be analysed via MMBMs due to the so-called fluid embedding technique (see, e.g. [17]). Since the transformation of a PH-MAP to an MMBM has been described extensively in other papers already (see, e.g. [7] and [17]), we do not wish to repeat this here and perform the analysis starting from a given MMBM. This also serves to simplify the notation. At suitable places we will remark on the relation to PH-MAPs.

The paper is structured as follows. Section 2 contains an exact definition of the problem to be analysed. In Section 3, the necessary preparatory results from recent literature are presented. Section 4 contains the main result, while examples and applications will be presented in the last section.

\section{The exit problem for reflected MMBMs}

Let $\mathcal{g}=\left(J_{t}: t \geq 0\right)$ denote an irreducible Markov process with a finite state space $E=$ $\{1, \ldots, m\}$ and infinitesimal generator matrix $Q=\left(q_{i j}\right)_{i, j \in E}$. We call $J_{t}$ the phase at time $t$ and $\mathcal{g}$ the phase process. Choosing parameters $\mu_{i} \in \mathbb{R}$ and $\sigma_{i} \geq 0$ for all $i \in E$, we define the level process $\mathcal{X}=\left(X_{t}: t \geq 0\right)$ by

$$
X_{t}=X_{0}+\int_{0}^{t} \mu_{J_{s}} \mathrm{~d} s+\int_{0}^{t} \sigma_{J_{s}} \mathrm{~d} W_{s}
$$

for all $t \geq 0$, where $\mathcal{W}=\left(W_{t}: t \geq 0\right)$ denotes a standard Wiener process that is independent of $\mathcal{g}$. Then $(\mathcal{X}, \mathcal{g})$ is called an MMBM. An MMBM is a MAP without jumps.

Some results for MMBMs go back to the 1990s, with Rogers [19] investigating Wiener-Hopf factorisation and stationary distributions for the case that $\sigma_{i}=\varepsilon$ is independent of the phase process. Around the same time, Asmussen [1] determined hitting probabilities and, based on these, expressions for the stationary distributions. More recent results are given in [10] and [14], in which MMBMs with two reflecting barriers are analysed. Some properties of scale functions for MMBMs are derived in [9] and [16]. Occupation times for MMBMs are investigated in [9].

Define the supremum process $\delta=\left(S_{t}: t \geq 0\right)$ by $S_{t}:=\sup _{s \leq t} X_{s} \vee 0$ for all $t \geq 0$, and the reflected process $\mathcal{y}:=s-\mathcal{X}$. For a fixed level $a>0$, let

$$
\sigma(a):=\inf \left\{t \geq 0: Y_{t} \geq a\right\} \quad \text { and } \quad \sigma_{-}(a):=\sup \left\{t \leq \sigma(a): Y_{t}=0\right\} .
$$


We shall seek to determine the joint distribution of $\sigma(a), \sigma_{-}(a)$, and $S_{\sigma(a)}$ in the form of the measure

$$
\mathrm{E}\left(\mathrm{e}^{-\alpha\left(\sigma(a)-\sigma_{-}(a)\right)} \mathrm{e}^{-\gamma \sigma_{-}(a)} ; S_{\sigma(a)} \in \mathrm{d} x\right),
$$

where $\alpha, \gamma, x \geq 0$. To be more precise, we shall obtain a finer result, namely an expression for

$$
\mathrm{E}\left(\exp \left[-\int_{0}^{\sigma_{-}(a)} r_{J_{s}} \mathrm{~d} s\right] \exp \left[-\int_{\sigma_{-}(a)}^{\sigma(a)} r_{J_{s}}^{\prime} \mathrm{d} s\right] ; S_{\sigma(a)} \in \mathrm{d} x\right)
$$

where $r_{i}, r_{i}^{\prime} \geq 0$ for all $i \in E$. The latter yields the joint distribution of the occupation times in each phase during the time intervals $\left[0, \sigma_{-}(a)\right]$ and $\left[\sigma_{-}(a), \sigma(a)\right]$; cf. [9] for occupation times of MMBMs.

Remark 1. A MAP $(\tilde{X}, \tilde{g})$ with phase-type jumps (PH-MAP) can be transformed into an MMBM as shown in detail in [7, Section 2.1]. The resulting MMBM has a phase space $E=E_{+} \cup E_{p} \cup E_{\sigma} \cup E_{n} \cup E_{-}$, where

$$
\begin{aligned}
& E_{p}=\left\{i \in \tilde{E}: \tilde{\mu}_{i}>0, \tilde{\sigma}_{i}=0\right\}, \\
& E_{n}=\left\{i \in \tilde{E}: \tilde{\mu}_{i}<0, \tilde{\sigma}_{i}=0\right\}, \\
& E_{\sigma}=\left\{i \in \tilde{E}: \tilde{\sigma}_{i}>0\right\},
\end{aligned}
$$

and phases in $E_{ \pm}$represent parts of the jumps (see [7, Section 2.1] for a precise definition). This method is called fluid embedding and has been described in Section 3 of [17], Section 2.7 of [15], and Section 2.2 of [7].

Let $\tilde{\mathcal{S}}$ be the supremum process of $\tilde{X}$. Furthermore, define the reflected process $\tilde{y}:=\tilde{\mathcal{S}}-\tilde{X}$ and $\tilde{\sigma}(a):=\inf \left\{t \geq 0: \tilde{Y}_{t} \geq a\right\}$ as well as $\tilde{\sigma}_{-}(a):=\sup \left\{t \leq \tilde{\sigma}(a): \tilde{Y}_{t}=0\right\}$ for a fixed level $a>0$. Define $\tilde{E}:=E_{p} \cup E_{\sigma} \cup E_{n}$. Then

$$
\tilde{\sigma}_{-}(a)=\int_{0}^{\sigma_{-}(a)} \mathbb{I}_{\left\{J_{s} \in \tilde{E}\right\}} \mathrm{d} s \quad \text { and } \quad \tilde{\sigma}(a)-\tilde{\sigma}_{-}(a)=\int_{\sigma_{-}(a)}^{\sigma(a)} \mathbb{I}_{\left\{J_{s} \in \tilde{E}\right\}} \mathrm{d} s,
$$

where $\mathbb{I}_{A}$ denotes the indicator function of an event $A$. Furthermore, $\tilde{S}_{\tilde{\sigma}(a)}=S_{\sigma(a)}$. Thus, we obtain

$$
\begin{aligned}
& \mathrm{E}\left(\mathrm{e}^{-\alpha\left(\tilde{\sigma}(a)-\tilde{\sigma}_{-}(a)\right)} \mathrm{e}^{-\gamma \tilde{\sigma}_{-}(a)} ; \tilde{S}_{\tilde{\sigma}(a)} \in \mathrm{d} x\right) \\
& \quad=\mathrm{E}\left(\exp \left[-\int_{0}^{\sigma_{-}(a)} r_{J_{s}} \mathrm{~d} s\right] \exp \left[-\int_{\sigma_{-}(a)}^{\sigma(a)} r_{J_{s}}^{\prime} \mathrm{d} s\right] ; S_{\sigma(a)} \in \mathrm{d} x\right)
\end{aligned}
$$

by setting $r_{i}:=\gamma, r_{i}^{\prime}:=\alpha$ for $i \in \tilde{E}$, and $r_{i}=r_{i}^{\prime}:=0$ for $i \in E_{ \pm}$. Hence, the joint distribution of $\tilde{\sigma}_{-}(a), \tilde{\sigma}(a)$, and $\tilde{S}_{\tilde{\sigma}(a)}$ can be obtained via the analysis of a suitable MMBM.

\section{Preliminaries: exit problems for free MMBMs}

We shall call $(\mathcal{X}, g)$ the free MMBM. In order to derive the desired result on exit times for the reflected process $\mathcal{Y}$, we need some results from the literature on exit times for the free MMBM. For ease of reference, they will be described in this section in a form that suits our later use of them. 


\subsection{The one-sided exit problem}

Define the first passage times $\tau(x):=\inf \left\{t \geq 0: X_{t}>x\right\}$ for all $x \geq 0$, and assume that $X_{0}=0$. Consider an $E$-dimensional row vector $\boldsymbol{r}=\left(r_{i}: i \in E\right)$ with nonnegative entries $r_{i} \geq 0$ for all $i \in E$. Define

$$
\mathrm{E}_{i j}\left(\exp \left[-\int_{0}^{\tau(x)} r_{J_{s}} \mathrm{~d} s\right]\right):=\mathrm{E}\left(\exp \left[-\int_{0}^{\tau(x)} r_{J_{s}} \mathrm{~d} s\right] ; J_{\tau(x)}=j \mid J_{0}=i, X_{0}=0\right)
$$

for $i, j \in E$ and $\mathrm{E}\left(\exp \left[-\int_{0}^{\tau(x)} r_{J_{s}} \mathrm{~d} s\right]\right)$ as the $(E \times E)$-matrix with these entries. The values $r_{i}$ may be interpreted as state-dependent killing rates; see Section 3 of [17]. We shall distinguish the phases by the subspaces

$$
E_{a}:=\left\{i \in E: \sigma_{i}>0 \text { or } \mu_{i}>0\right\} \quad \text { and } \quad E_{d}:=E \backslash E_{a},
$$

where phases in $E_{a}$ and $E_{d}$ are respectively called ascending and descending. The same arguments as in [6, Section 3] yield

$$
\mathrm{E}_{(d, d)}\left(\exp \left[-\int_{0}^{\tau(x)} r_{J_{s}} \mathrm{~d} s\right]\right)=\mathbf{0} \quad \text { and } \quad \mathrm{E}_{(a, d)}\left(\exp \left[-\int_{0}^{\tau(x)} r_{J_{s}} \mathrm{~d} s\right]\right)=\mathbf{0},
$$

where $\mathbf{0}$ denotes a zero matrix of suitable dimension. Furthermore,

$$
\begin{aligned}
& \mathrm{E}_{(d, a)}\left(\exp \left[-\int_{0}^{\tau(x)} r_{J_{s}} \mathrm{~d} s\right]\right)=A(\boldsymbol{r}) \mathrm{e}^{U(\boldsymbol{r}) x} \\
& \text { and } \mathrm{E}_{(a, a)}\left(\exp \left[-\int_{0}^{\tau(x)} r_{J_{s}} \mathrm{~d} s\right]\right)=\mathrm{e}^{U(\boldsymbol{r}) x}
\end{aligned}
$$

where the matrices $A(\boldsymbol{r})$ and $U(\boldsymbol{r})$ can be computed as follows. For arguments $\beta \geq 0$, define the functions $\phi_{i}(\beta):=\beta / \mu_{i}$ for $i \in E_{a}$ with $\sigma_{i}=0$ as well as

$$
\phi_{i}(\beta)=\frac{1}{\sigma_{i}} \sqrt{2 \beta+\frac{\mu_{i}^{2}}{\sigma_{i}^{2}}}-\frac{\mu_{i}}{\sigma_{i}^{2}} \text { and } \phi_{i}^{*}(\beta)=\frac{1}{\sigma_{i}} \sqrt{2 \beta+\frac{\mu_{i}^{2}}{\sigma_{i}^{2}}}+\frac{\mu_{i}}{\sigma_{i}^{2}}
$$

for $i \in E_{a}$ with $\sigma_{i}>0$. The iteration to determine $A(\boldsymbol{r})$ and $U(\boldsymbol{r})$ is slightly changed from [7, Section 2.2] to the following form. We obtain $(A(\boldsymbol{r}), U(\boldsymbol{r}))=\lim _{n \rightarrow \infty}\left(A_{n}, U_{n}\right)$ for initial values $A_{0}:=\mathbf{0}, U_{0}:=-\operatorname{diag}\left(\phi_{i}\left(q_{i}+r_{i}\right)\right)_{i \in E_{a}}$, and iterations

$$
e_{i}^{\prime} U_{n+1}=-\frac{q_{i}+r_{i}}{\mu_{i}} e_{i}^{\prime}+\frac{1}{\mu_{i}} \sum_{j \in E, j \neq i} q_{i j} e_{j}^{\prime}\left(\begin{array}{c}
I_{a} \\
A_{n}
\end{array}\right)
$$

for $i \in E_{a}$ and $\sigma_{i}=0$,

$$
e_{i}^{\prime} U_{n+1}=-\phi_{i}\left(q_{i}+r_{i}\right) e_{i}^{\prime}+\frac{2}{\sigma_{i}^{2}} \sum_{j \in E, j \neq i} q_{i j} e_{j}^{\prime}\left(\begin{array}{c}
I_{a} \\
A_{n}
\end{array}\right)\left(\phi_{i}^{*}\left(q_{i}+r_{i}\right) I-U_{n}\right)^{-1}
$$

for $\sigma_{i}>0$, and

$$
e_{i}^{\prime} A_{n+1}=\sum_{j \in E, j \neq i} q_{i j} e_{j}^{\prime}\left(\begin{array}{c}
I_{a} \\
A_{n}
\end{array}\right)\left(\left(q_{i}+r_{i}\right) I+\mu_{i} U_{n}\right)^{-1}
$$

for $i \in E_{d}$. Here $e_{i}^{\prime}$ denotes the $i$ th canonical row base vector, $q_{i}:=-q_{i i}$ for all $i \in E$, and $I_{a}$ is the identity matrix on $E_{a}$. The case $\boldsymbol{r}=\mathbf{0}$ has been analysed earlier in [1]. 


\subsection{The two-sided exit problem}

Denote the exit time of $\mathcal{X}$ from the interval $[l, u]$ by $\tau(l, u):=\inf \left\{t \geq 0: X_{t} \notin[l, u]\right\}$, where $l<u$. Choose a positive vector $\boldsymbol{r}:=\left(r_{i}: i \in E\right)$, i.e. $r_{i} \geq 0$ for all $i \in E$ and $\|\boldsymbol{r}\|:=\sum_{i \in E}\left|r_{i}\right|>0$. Define

$$
\Psi_{i j}^{+}(l, u \mid x):=\mathrm{E}\left(\exp \left[-\int_{0}^{\tau(l, u)} r_{J_{s}} \mathrm{~d} s\right] ; X_{\tau(l, u)}=u, J_{\tau(l, u)}=j \mid J_{0}=i, X_{0}=x\right),
$$

where $x \in[l, u]$ and $i, j \in E$, and define the matrix $\Psi_{r}^{+}(l, u \mid x):=\left(\Psi_{i j}^{+}(l, u \mid x)\right)_{i, j \in E}$. A formula for $\Psi_{r}^{+}(l, u \mid x)$ has been derived in [17]. In order to state it, we need some additional notation. In order to simplify this notation, we shall from now on exclude the case of a phase $i \in E$ with $\mu_{i}=\sigma_{i}=0$.

Let $\left(\mathcal{X}^{+}, \mathcal{g}\right)$ denote the original MMBM, and define the process $\left(\mathcal{X}^{-}, \mathcal{g}\right) \stackrel{\mathrm{D}}{=}\left(-\mathcal{X}^{+}, \mathcal{g}\right)$, where $\stackrel{\text { D }}{=}$ denotes equality in distribution. The two processes have the same generator matrix $Q$ for $g$, but the drift parameters are different. Denoting variation and drift parameters for $\chi^{ \pm}$ by $\sigma_{i}^{ \pm}$and $\mu_{i}^{ \pm}$, respectively, this means that $\sigma_{i}^{-}=\sigma_{i}^{+}$and $\mu_{i}^{-}=-\mu_{i}^{+}$for all $i \in E$. Since we have excluded a phase $i \in E$ with $\mu_{i}=\sigma_{i}=0$, the ascending phases of $\left(\mathcal{X}^{+}, \mathcal{g}\right)$ are descending for $\left(\mathcal{X}^{-}, \mathcal{g}\right)$ and vice versa.

Let $A^{ \pm}(\boldsymbol{r})$ and $U^{ \pm}(\boldsymbol{r})$ denote the matrices that determine the first passage times of $\chi^{ \pm}$in (1). We shall write $A^{ \pm}=A^{ \pm}(\boldsymbol{r})$ and $U^{ \pm}=U^{ \pm}(\boldsymbol{r})$ if we do not wish to underline the dependence on $\boldsymbol{r}$. Let $I_{\sigma}$ denote the identity matrix on $E_{\sigma}:=\left\{i \in E: \sigma_{i}>0\right\}$, and define the matrices

$$
C^{+}:=C^{+}(\boldsymbol{r}):=\left(\begin{array}{ll}
\mathbf{0} & I_{\sigma} \\
A^{+}(\boldsymbol{r})
\end{array}\right) \quad \text { and } \quad C^{-}:=C^{-}(\boldsymbol{r}):=\left(\begin{array}{cr}
A^{-}(\boldsymbol{r}) \\
I_{\sigma} & \mathbf{0}
\end{array}\right)
$$

of dimensions $\left(E_{\sigma} \cup E_{d}\right) \times E_{a}$ and $E_{a} \times\left(E_{\sigma} \cup E_{d}\right)$, respectively. Furthermore, define

$$
W^{+}:=W^{+}(\boldsymbol{r}):=\left(\begin{array}{c}
I_{a} \\
A^{+}(\boldsymbol{r})
\end{array}\right) \quad \text { and } \quad W^{-}:=W^{-}(\boldsymbol{r}):=\left(\begin{array}{cc}
A^{-}(\boldsymbol{r}) \\
I_{\sigma} & \mathbf{0} \\
\mathbf{0} & I_{d}
\end{array}\right),
$$

which are matrices of dimensions $E \times E_{a}$ and $E \times\left(E_{\sigma} \cup E_{d}\right)$. Finally, let $Z^{ \pm}:=C^{ \pm} \mathrm{e}^{U^{ \pm} \cdot(u-l)}$. Then Equation (23) of [17] states that

$$
\Psi_{r}^{+}(l, u \mid x)=\left(W^{+} \mathrm{e}^{U^{+} \cdot(u-x)}-W^{-} \mathrm{e}^{U^{-} \cdot(x-l)} C^{+} \mathrm{e}^{U^{+} \cdot(u-l)}\right)\left(I-Z^{-} Z^{+}\right)^{-1}
$$

for $l \leq x \leq u$. By reflection at the initial level $x$, we obtain, from (2),

$$
\begin{aligned}
\Psi_{r}^{-}(l, u \mid x) & :=\mathrm{E}\left(\exp \left[-\int_{0}^{\tau(l, u)} r_{J_{s}} \mathrm{~d} s\right] ; X_{\tau(l, u)}=l \mid X_{0}=x\right) \\
& =\left(W^{-} \mathrm{e}^{U^{-} \cdot(x-l)}-W^{+} \mathrm{e}^{U^{+} \cdot(u-x)} C^{-} \mathrm{e}^{U^{-} \cdot(u-l)}\right)\left(I-Z^{+} Z^{-}\right)^{-1}
\end{aligned}
$$

for $l \leq x \leq u$. Note that the expressions in (2) and (3) depend on a choice of $\boldsymbol{r}$.

Remark 2. Noting that $\|\boldsymbol{r}\|>0$ we first see that $Z^{-}$and $Z^{+}$have row sums strictly less than 1 , which implies that $\left(I-Z^{-} Z^{+}\right)$is invertible. Furthermore, $\left(I-Z^{-} Z^{+}\right)^{-1}=\sum_{n=0}^{\infty}\left(Z^{-} Z^{+}\right)^{n}$ and $Z^{-} Z^{+}$represents a crossing of the interval $[0, b]$ from $b$ to 0 and back. Hence, (2) has a clear probabilistic interpretation. The term $W^{+} \mathrm{e}^{U^{+} \cdot(b-x)}$ simply yields the event that $\mathcal{X}$ exits from $b$ (before an exponential time of parameter $\gamma$ ). The correction term $W^{-} \mathrm{e}^{U^{-} \cdot x} Z^{+}$refers 
to the event that $\mathcal{X}$ descends below 0 before exiting from $b$. Multiplication by $\left(I-Z^{-} Z^{+}\right)^{-1}$ yields all possible combinations with any number of subsequent (down and up) crossings over the complete interval $[0, b]$.

Note that, for $\boldsymbol{r}=\mathbf{0}$ and $\mathcal{X}$ having a zero-mean drift, the matrix $I-Z^{-} Z^{+}$may be singular. This is the reason why we postulate $\boldsymbol{r}$ to be strictly positive.

Remark 3. Since $Z^{+}=C^{+} \mathrm{e}^{U^{+} \cdot b}$, we can write $\Psi_{\boldsymbol{r}}^{+}(0, b \mid x)$ in the form

$$
\Psi_{r}^{+}(0, b \mid x)=\left(W^{+} \mathrm{e}^{-U^{+} \cdot x}-W^{-} \mathrm{e}^{U^{-} \cdot x} C^{+}\right)\left(\mathrm{e}^{-U^{+} \cdot b}-C^{-} \mathrm{e}^{U^{-} \cdot b} C^{+}\right)^{-1} .
$$

This comes closer to the usual expression of the exit time distribution in terms of scale functions. For instance, let $\mathcal{X}$ be a Brownian motion with variation $\sigma>0$ and drift $\mu \in \mathbb{R}$. Set $\boldsymbol{r}:=\gamma$, which is a real number as $E=E_{\sigma}$ contains a single phase only. For the same reason, $W^{ \pm}=$ $C^{ \pm}=1$ and $A^{ \pm}$are void. Furthermore,

$$
U^{ \pm}=\frac{ \pm \mu-\sqrt{\mu^{2}+2 \gamma \sigma^{2}}}{\sigma^{2}} .
$$

Define $r:=-U^{+}$and $s:=U^{-}$. Then

$$
\Psi_{\gamma}^{+}(0, b \mid x)=\frac{\mathrm{e}^{r x}-\mathrm{e}^{s x}}{\mathrm{e}^{r b}-\mathrm{e}^{s b}}
$$

(cf. [13, Equations (2.12)-(2.15)], where the $\gamma$-scale function is given as $g(x)=\mathrm{e}^{r x}-\mathrm{e}^{s x}$.

\section{Main result}

Let $\boldsymbol{r}=\left(r_{i}: i \in E\right)$ and $\boldsymbol{r}^{\prime}=\left(r_{i}^{\prime}: i \in E\right)$ with $r_{i}, r_{i}^{\prime} \geq 0$ for all $i \in E$ denote two exit rate vectors. In light of Remark 2 we will from now on assume that $\|\boldsymbol{r}\|>0$ and $\left\|\boldsymbol{r}^{\prime}\right\|>0$.

Theorem 1. The joint distribution of $\sigma(a), \sigma_{-}(a)$, and $S_{\sigma(a)}$ is given by

$$
\begin{gathered}
\mathrm{E}\left(\exp \left[-\int_{0}^{\sigma_{-}(a)} r_{J_{s}} \mathrm{~d} s\right] \exp \left[-\int_{\sigma_{-}(a)}^{\sigma(a)} r_{J_{s}}^{\prime} \mathrm{d} s\right] ; S_{\sigma(a)} \in \mathrm{d} x \mid X_{0}=0\right) \\
=\Psi_{\boldsymbol{r}}^{+}(0, a \mid a) \mathrm{e}^{G_{\boldsymbol{r}}(a) x} H_{\boldsymbol{r}^{\prime}}(a) \mathrm{d} x
\end{gathered}
$$

for $x>0$, where

$$
\begin{aligned}
G_{\boldsymbol{r}}(a)= & \left(U^{+}(\boldsymbol{r}) \mathrm{e}^{-U^{+}(\boldsymbol{r}) a}+C^{-}(\boldsymbol{r}) \mathrm{e}^{U^{-}(\boldsymbol{r}) a} U^{-}(\boldsymbol{r}) C^{+}(\boldsymbol{r})\right) \\
& \times\left(\mathrm{e}^{-U^{+}(\boldsymbol{r}) a}-C^{-}(\boldsymbol{r}) \mathrm{e}^{U^{-}(\boldsymbol{r}) a} C^{+}(\boldsymbol{r})\right)^{-1}
\end{aligned}
$$

and

$$
H_{\boldsymbol{r}^{\prime}}(a)=\left(C^{-}\left(\boldsymbol{r}^{\prime}\right) U^{-}\left(\boldsymbol{r}^{\prime}\right)+U^{+}\left(\boldsymbol{r}^{\prime}\right) C^{-}\left(\boldsymbol{r}^{\prime}\right)\right)\left(C^{+}\left(\boldsymbol{r}^{\prime}\right) \mathrm{e}^{U^{+}\left(\boldsymbol{r}^{\prime}\right) a} C^{-}\left(\boldsymbol{r}^{\prime}\right)-\mathrm{e}^{-U^{-}\left(\boldsymbol{r}^{\prime}\right) a}\right)^{-1} .
$$

There is further (for $J_{0} \in E_{d}$ ) an atom at $S_{\sigma(a)}=0$ with

$$
\mathrm{E}\left(\exp \left[-\int_{0}^{\sigma(a)}{ } r_{J_{s}} \mathrm{~d} s\right] ; S_{\sigma(a)}=0, \sigma_{-}(a)=0 \mid X_{0}=0\right)=\Psi_{\boldsymbol{r}}^{-}(0, a \mid a) .
$$


Proof. Assume that $X_{0}=0$, i.e. $S_{0}=0$. We consider the sequence $\left(T_{n}(\varepsilon): n \in \mathbb{N}_{0}\right)$ of stopping times defined by $T_{0}(\varepsilon):=0$ and

$$
T_{n}(\varepsilon):=\inf \left\{t \geq T_{n-1}(\varepsilon): S_{t}=n \varepsilon, \min _{T_{n-1}(\varepsilon) \leq s \leq t} X_{s}>(n-1) \varepsilon-a\right\},
$$

where inf $\varnothing:=\infty$. The times $T_{n+1}(\varepsilon)-T_{n}(\varepsilon), n \in \mathbb{N}_{0}$, are conditionally independent given the phase process $g$. On the set $\left\{T_{n}(\varepsilon)<\infty\right\}$, we observe that $X_{T_{n}(\varepsilon)}=S_{T_{n}(\varepsilon)}=n \varepsilon$ for any $n \in \mathbb{N}_{0}$. Thus, the (generalised) Laplace transform of $T_{n+1}(\varepsilon)-T_{n}(\varepsilon)$ with argument $\boldsymbol{r}$ is given by $\Psi_{r}^{+}(0, a+\varepsilon \mid a)$ and, hence, independent of $n \in \mathbb{N}_{0}$. Furthermore, $J_{T_{n}(\varepsilon)} \in E_{a}$ for all $n \in \mathbb{N}$. Thus,

$$
\mathrm{E}\left(\exp \left[-\int_{0}^{T_{n}(\varepsilon)}{ }{ }_{J_{s}} \mathrm{~d} s\right] ; T_{n}(\varepsilon)<\infty\right)=\Psi_{\boldsymbol{r}}^{+}(0, a+\varepsilon \mid a)\left(\Psi_{\boldsymbol{r}}^{+}(0, a+\varepsilon \mid a)_{(a, a)}\right)^{n-1}
$$

for $n \in \mathbb{N}$ and $\boldsymbol{r}=\left(r_{i}: i \in E\right) \geq \mathbf{0}$ (entrywise), where $\Psi_{\boldsymbol{r}}^{+}(0, a+\varepsilon \mid a)_{(a, a)}$ denotes the upper $E_{a} \times E_{a}$ block of the matrix

$$
\Psi_{r}^{+}(0, a+\varepsilon \mid a)=\left(\begin{array}{c}
\Psi_{r}^{+}(0, a+\varepsilon \mid a)_{(a, a)} \\
\Psi_{r}^{+}(0, a+\varepsilon \mid a)_{(d, a)}
\end{array}\right)
$$

referring to ascending initial phases. Thus,

$$
\begin{aligned}
\Psi_{\boldsymbol{r}}^{+}(0, a+\varepsilon \mid a)_{(a, a)}= & \left(\mathrm{e}^{U^{+}(\boldsymbol{r}) \varepsilon}-C^{-}(\boldsymbol{r}) \mathrm{e}^{U^{-}(\boldsymbol{r}) a} C^{+}(\boldsymbol{r}) \mathrm{e}^{U^{+}(\boldsymbol{r})(a+\varepsilon)}\right) \\
& \times\left(I_{a}-C^{-}(\boldsymbol{r}) \mathrm{e}^{U^{-}(\boldsymbol{r})(a+\varepsilon)} C^{+}(\boldsymbol{r}) \mathrm{e}^{U^{+}(\boldsymbol{r})(a+\varepsilon)}\right)^{-1},
\end{aligned}
$$

according to (2). The probabilities of failure for this matrix-geometric distribution are again independent of $n \in \mathbb{N}$ and given by $\mathrm{P}\left(X_{\tau(0, a+\varepsilon)}=0 \mid X_{0}=a\right)$. The respective generalised Laplace transform with argument $\boldsymbol{r}^{\prime}$ is the upper block of

$$
\begin{aligned}
\mathrm{E}\left(\exp \left[-\int_{0}^{\tau(0, a+\varepsilon)}{ }^{\prime}{ }_{J_{s}}^{\prime} \mathrm{d} s\right] ; X_{\tau(0, a+\varepsilon)}=0 \mid X_{0}=a\right) & =\Psi_{\boldsymbol{r}^{\prime}}^{-}(0, a+\varepsilon \mid a) \\
& =\left(\begin{array}{c}
\Psi_{\boldsymbol{r}^{\prime}}^{-}(0, a+\varepsilon \mid a)_{(a, \sigma \cup d)} \\
\Psi_{\boldsymbol{r}^{\prime}}^{-}(0, a+\varepsilon \mid a)_{(d, \sigma \cup d)}
\end{array}\right) .
\end{aligned}
$$

According to (3),

$$
\begin{aligned}
\Psi_{\boldsymbol{r}^{\prime}}^{-}(0, a+\varepsilon \mid a)_{(a, \sigma \cup d)}= & \left(C^{-}\left(\boldsymbol{r}^{\prime}\right) \mathrm{e}^{U^{-}\left(\boldsymbol{r}^{\prime}\right) a}-\mathrm{e}^{U^{+}\left(\boldsymbol{r}^{\prime}\right) \varepsilon} C^{-}\left(\boldsymbol{r}^{\prime}\right) \mathrm{e}^{U^{-}\left(\boldsymbol{r}^{\prime}\right)(a+\varepsilon)}\right) \\
& \times\left(I_{\sigma \cup d}-C^{+}\left(\boldsymbol{r}^{\prime}\right) \mathrm{e}^{U^{+}\left(\boldsymbol{r}^{\prime}\right)(a+\varepsilon)} C^{-}\left(\boldsymbol{r}^{\prime}\right) \mathrm{e}^{U^{-}\left(\boldsymbol{r}^{\prime}\right)(a+\varepsilon)}\right)^{-1} \\
= & \left(C^{-}\left(\boldsymbol{r}^{\prime}\right) \mathrm{e}^{-U^{-}\left(\boldsymbol{r}^{\prime}\right) \varepsilon}-\mathrm{e}^{U^{+}\left(\boldsymbol{r}^{\prime}\right) \varepsilon} C^{-}\left(\boldsymbol{r}^{\prime}\right)\right) \\
& \times\left(\mathrm{e}^{-U^{-}\left(\boldsymbol{r}^{\prime}\right)(a+\varepsilon)}-C^{+}\left(\boldsymbol{r}^{\prime}\right) \mathrm{e}^{U^{+}\left(\boldsymbol{r}^{\prime}\right)(a+\varepsilon)} C^{-}\left(\boldsymbol{r}^{\prime}\right)\right)^{-1}
\end{aligned}
$$

where $I_{\sigma \cup d}$ denotes the identity matrix on $E_{\sigma} \cup E_{d}$. Let $N:=\max \left\{n \in \mathbb{N}_{0}: T_{n}(\varepsilon)<\infty\right\}$ and $S(\varepsilon):=\inf \left\{t>T_{N}(\varepsilon): X_{t}<N \varepsilon-a\right\}$. Then we can write

$$
\begin{aligned}
& \mathrm{E}\left(\exp \left[-\int_{0}^{T_{n}(\varepsilon)} r_{J_{s}} \mathrm{~d} s\right] \exp \left[-\int_{T_{n}(\varepsilon)}^{S(\varepsilon)} r_{J_{s}}^{\prime} \mathrm{d} s\right] ; N=n\right) \\
& \quad=\Psi_{\boldsymbol{r}}^{+}(0, a+\varepsilon \mid a)\left(\Psi_{\boldsymbol{r}}^{+}(0, a+\varepsilon \mid a)_{(a, a)}\right)^{n-1} \Psi_{\boldsymbol{r}^{\prime}}^{-}(0, a+\varepsilon \mid a)_{(a, \sigma \cup d)} .
\end{aligned}
$$


Now letting $\varepsilon \rightarrow 0$ and $n \rightarrow \infty$ such that $n \varepsilon \rightarrow x$, we obtain

$$
\begin{aligned}
& \mathrm{E}\left(\exp \left[-\int_{0}^{T_{n}(\varepsilon)} r_{J_{s}} \mathrm{~d} s\right] \exp \left[-\int_{T_{n}(\varepsilon)}^{S(\varepsilon)} r_{J_{s}}^{\prime} \mathrm{d} s\right] ; N=n\right) \\
& \rightarrow \mathrm{E}\left(\exp \left[-\int_{0}^{\sigma_{-}(a)} r_{J_{s}} \mathrm{~d} s\right] \exp \left[-\int_{\sigma_{-}(a)}^{\sigma(a)} r_{J_{s}}^{\prime} \mathrm{d} s\right] ; S_{\sigma(a)} \in \mathrm{d} x\right) .
\end{aligned}
$$

The latter has a defective matrix-exponential distribution with parameters

$$
G_{\boldsymbol{r}}(a)=\lim _{\varepsilon \downarrow 0} \frac{1}{\varepsilon}\left(\Psi_{\boldsymbol{r}}^{+}(0, a+\varepsilon \mid a)_{(a, a)}-I_{a}\right)
$$

and

$$
H_{\boldsymbol{r}^{\prime}}(a)=\lim _{\varepsilon \downarrow 0} \frac{1}{\varepsilon}\left(\Psi_{\boldsymbol{r}^{\prime}}^{-}(0, a+\varepsilon \mid a)_{(a, \sigma \cup d)}-\mathbf{0}\right) .
$$

For the first parameter, we obtain, from (2),

$$
\begin{aligned}
& G_{\boldsymbol{r}}(a)=\lim _{\varepsilon \downarrow 0} \frac{1}{\varepsilon}\left(\left(\mathrm{e}^{U^{+}(\boldsymbol{r}) \varepsilon}-C^{-}(\boldsymbol{r}) \mathrm{e}^{U^{-}(\boldsymbol{r}) a} C^{+}(\boldsymbol{r}) \mathrm{e}^{U^{+}(\boldsymbol{r})(a+\varepsilon)}\right)\right. \\
&\left.-\left(I_{a}-C^{-}(\boldsymbol{r}) \mathrm{e}^{U^{-}(\boldsymbol{r})(a+\varepsilon)} C^{+}(\boldsymbol{r}) \mathrm{e}^{U^{+}(\boldsymbol{r})(a+\varepsilon)}\right)\right) \\
& \quad \times\left(I_{a}-C^{-}(\boldsymbol{r}) \mathrm{e}^{U^{-}(\boldsymbol{r})(a+\varepsilon)} C^{+}(\boldsymbol{r}) \mathrm{e}^{U^{+}(\boldsymbol{r})(a+\varepsilon)}\right)^{-1} \\
&=\lim _{\varepsilon \downarrow 0} \frac{1}{\varepsilon}\left(\mathrm{e}^{U^{+}(\boldsymbol{r}) \varepsilon}-I_{a}+C^{-}(\boldsymbol{r}) \mathrm{e}^{U^{-}(\boldsymbol{r}) a}\left(\mathrm{e}^{U^{-}(\boldsymbol{r}) \varepsilon}-I_{\sigma \cup d}\right) C^{+}(\boldsymbol{r}) \mathrm{e}^{U^{+}(\boldsymbol{r})(a+\varepsilon)}\right) \\
& \quad \times\left(I_{a}-C^{-}(\boldsymbol{r}) \mathrm{e}^{U^{-}(\boldsymbol{r}) a} C^{+}(\boldsymbol{r}) \mathrm{e}^{U^{+}(\boldsymbol{r}) a}\right)^{-1} \\
&=\left(U^{+}(\boldsymbol{r})+C^{-}(\boldsymbol{r}) \mathrm{e}^{U^{-}(\boldsymbol{r}) a} U^{-}(\boldsymbol{r}) C^{+}(\boldsymbol{r}) \mathrm{e}^{U^{+}(\boldsymbol{r}) a}\right) \\
& \times\left(I_{a}-C^{-}(\boldsymbol{r}) \mathrm{e}^{U^{-}(\boldsymbol{r}) a} C^{+}(\boldsymbol{r}) \mathrm{e}^{U^{+}(\boldsymbol{r}) a}\right)^{-1} \\
&=\left(U^{+}(\boldsymbol{r}) \mathrm{e}^{-U^{+}(\boldsymbol{r}) a}+C^{-}(\boldsymbol{r}) \mathrm{e}^{U^{-}(\boldsymbol{r}) a} U^{-}(\boldsymbol{r}) C^{+}(\boldsymbol{r})\right) \\
& \times\left(\mathrm{e}^{-U^{+}(\boldsymbol{r}) a}-C^{-}(\boldsymbol{r}) \mathrm{e}^{U^{-}(\boldsymbol{r}) a} C^{+}(\boldsymbol{r})\right)^{-1},
\end{aligned}
$$

while the second parameter is determined from (3) as

$$
\begin{aligned}
H_{\boldsymbol{r}^{\prime}}(a)= & \lim _{\varepsilon \downarrow 0} \frac{1}{\varepsilon}\left(C^{-}\left(\boldsymbol{r}^{\prime}\right) \mathrm{e}^{-U^{-}\left(\boldsymbol{r}^{\prime}\right) \varepsilon}-\mathrm{e}^{U^{+}\left(\boldsymbol{r}^{\prime}\right) \varepsilon} C^{-}\left(\boldsymbol{r}^{\prime}\right)\right) \\
& \times\left(\mathrm{e}^{-U^{-}\left(\boldsymbol{r}^{\prime}\right)(a+\varepsilon)}-C^{+}\left(\boldsymbol{r}^{\prime}\right) \mathrm{e}^{U^{+}\left(\boldsymbol{r}^{\prime}\right)(a+\varepsilon)} C^{-}\left(\boldsymbol{r}^{\prime}\right)\right)^{-1} \\
= & \left(C^{-}\left(\boldsymbol{r}^{\prime}\right) U^{-}\left(\boldsymbol{r}^{\prime}\right)+U^{+}\left(\boldsymbol{r}^{\prime}\right) C^{-}\left(\boldsymbol{r}^{\prime}\right)\right)\left(C^{+}\left(\boldsymbol{r}^{\prime}\right) \mathrm{e}^{U^{+}\left(\boldsymbol{r}^{\prime}\right) a} C^{-}\left(\boldsymbol{r}^{\prime}\right)-\mathrm{e}^{-U^{-}\left(\boldsymbol{r}^{\prime}\right) a}\right)^{-1} .
\end{aligned}
$$

This proves the first statement. The statement about the atom $S_{\sigma(a)}=0$ is immediate.

\section{Examples and applications}

Example 1. The example given in Remark 3 of a Brownian motion fluid flow is explicated in Section 6 of [20]. There it is stated that

$$
\mathrm{E}\left(\mathrm{e}^{-\alpha \sigma(a)-\beta S_{\sigma(a)}-\gamma \sigma_{-}(a)}\right)=\frac{2 \mathrm{e}^{-2 a m} W^{(\alpha+\gamma)}(a)}{W^{(\alpha)}(a)\left(W^{(\alpha+\gamma)^{\prime}}(a)+\beta W^{(\alpha+\gamma)}(a)\right)}
$$

for $\sigma^{2}=1$ and $\mu=m$, noting that the notation $\sigma(a)$ and $\sigma_{-}(a)$ are replaced in [20] by $\tau(a)$ 
and $\tau_{-}(a)$, respectively. The scale function is therein defined as

$$
W^{(\alpha)}(a)=\frac{\mathrm{e}^{-a\left(m-\sqrt{m^{2}+2 \alpha}\right)}-\mathrm{e}^{-a\left(m+\sqrt{m^{2}+2 \alpha}\right)}}{\sqrt{m^{2}+2 \alpha}} .
$$

We now wish to arrive at the same expression via Theorem 1 . Since there is only one phase and $E=E_{\sigma}$, we find that $W^{ \pm}=C^{ \pm}=1$ and $A^{ \pm}$are void. Set $\boldsymbol{r}^{\prime}:=\alpha$ and $\boldsymbol{r}:=\alpha+\gamma$, which are positive real numbers now. For any $\beta>0$, we obtain $\Psi_{\beta}^{+}(a, a)=1$ and

$$
U^{ \pm}(\beta)= \pm m-\sqrt{m^{2}+2 \beta}
$$

for $\sigma^{2}=1$ and $\mu=m$, i.e. the $U^{ \pm}(\beta)$ are negative real numbers. This implies that

$$
G_{\alpha+\gamma}(a)=\frac{U^{+}(\alpha+\gamma) \mathrm{e}^{-U^{+}(\alpha+\gamma) a}+\mathrm{e}^{U^{-}(\alpha+\gamma) a} U^{-}(\alpha+\gamma)}{\mathrm{e}^{-U^{+}(\alpha+\gamma) a}-\mathrm{e}^{U^{-}(\alpha+\gamma) a}}
$$

and

$$
H_{\alpha}(a)=\frac{U^{-}(\alpha)+U^{+}(\alpha)}{\mathrm{e}^{U^{+}(\alpha) a}-\mathrm{e}^{-U^{-}(\alpha) a}}
$$

We begin by observing that

$$
\mathrm{E}\left(\mathrm{e}^{-\alpha \sigma(a)-\beta S_{\sigma(a)}-\gamma \sigma_{-}(a)}\right)=\int_{0}^{\infty} \mathrm{e}^{G_{\alpha+\gamma}(a) x} \mathrm{e}^{-\beta x} \mathrm{~d} x H_{\alpha}(a)=\left(\beta-G_{\alpha+\gamma}(a)\right)^{-1} H_{\alpha}(a) .
$$

Equations (5) and (6) yield

$$
W^{(\alpha)}(a)=\frac{\mathrm{e}^{-U^{+}(\alpha) a}-\mathrm{e}^{U^{-}(\alpha) a}}{\sqrt{m^{2}+2 \alpha}} \quad \text { and } \quad G_{\alpha+\gamma}(a)=-\frac{W^{(\alpha+\gamma)^{\prime}}(a)}{W^{(\alpha+\gamma)}(a)},
$$

as well as

$$
H_{\alpha}(a)=\frac{2 \sqrt{m^{2}+2 \alpha} \mathrm{e}^{-2 m a}}{\mathrm{e}^{-U^{+}(\alpha) a}-\mathrm{e}^{U^{-}(\alpha) a}}=\frac{2 \mathrm{e}^{-2 m a}}{W^{(\alpha)}(a)} .
$$

Hence, we obtain

$$
\mathrm{E}\left(\mathrm{e}^{-\alpha \sigma(a)-\beta S_{\sigma(a)}-\gamma \sigma_{-}(a)}\right)=\frac{W^{(\alpha+\gamma)}(a)}{W^{(\alpha+\gamma)^{\prime}}(a)+\beta W^{(\alpha+\gamma)}(a)} \frac{2 \mathrm{e}^{-2 m a}}{W^{(\alpha)}(a)},
$$

which is the same expression as (4).

Remark 4. Defining an $\boldsymbol{r}$-scale function for MMBMs by

$$
W_{\boldsymbol{r}}(x):=\mathrm{e}^{-U^{+}(\boldsymbol{r}) x}-C^{-}(\boldsymbol{r}) \mathrm{e}^{U^{-}(\boldsymbol{r}) x} C^{+}(\boldsymbol{r})
$$

for $x>0$, we see first that $G_{\boldsymbol{r}}(a)=-W_{\boldsymbol{r}}^{\prime}(a)\left[W_{\boldsymbol{r}}(a)\right]^{-1}$, where $W_{\boldsymbol{r}}^{\prime}(a)$ denotes the derivative of the function $W_{r}(x)$ at $x=a$.

In applications to insurance risk, a popular goal is to determine the expected amount of $\boldsymbol{r}$-discounted dividends paid before ruin. If the initial risk reserve is $u \geq 0$ and dividends are 
paid above a constant barrier of $a \geq u$, then the mean discounted dividends paid out before ruin can be computed as

$$
\begin{aligned}
V_{\boldsymbol{r}}(a \mid u):= & \Psi_{\boldsymbol{r}}^{+}(0, a \mid u) \mathrm{E}\left(S_{\sigma(a)} \exp \left[-\int_{0}^{\sigma_{-}(a)} r_{J_{s}} \mathrm{~d} s\right]\right) \\
= & \Psi_{\boldsymbol{r}}^{+}(0, a \mid u) \int_{0}^{\infty} \mathrm{e}^{G_{\boldsymbol{r}}(a) x} \mathrm{~d} x \\
= & \Psi_{\boldsymbol{r}}^{+}(0, a \mid u)\left[-G_{\boldsymbol{r}}(a)\right]^{-1} \\
= & \left(W^{+}(\boldsymbol{r}) \mathrm{e}^{-U^{+}(\boldsymbol{r}) u}-W^{-}(\boldsymbol{r}) \mathrm{e}^{U^{-}(\boldsymbol{r}) u} C^{+}(\boldsymbol{r})\right) \\
& \times\left(-U^{+}(\boldsymbol{r}) \mathrm{e}^{-U^{+}(\boldsymbol{r}) a}-C^{-}(\boldsymbol{r}) U^{-}(\boldsymbol{r}) \mathrm{e}^{U^{-}(\boldsymbol{r}) a} C^{+}(\boldsymbol{r})\right)^{-1}
\end{aligned}
$$

The term $\Psi_{r}^{+}(0, a \mid u)$ in the first equality includes the probability that the risk reserve reaches the barrier before ruin occurs. The probability of receiving no dividends before ruin is given by $\Psi_{0}^{-}(0, a \mid u)$. Since the time $\sigma_{-}(a)$ of the last dividend payment before ruin is a good time for stakeholders to pull out their investment, the discounting of the dividends is computed towards this time, which explains the term $\exp \left[-\int_{0}^{\sigma_{-}(a)} r_{J_{s}} \mathrm{~d} s\right]$ in the expectation.

Example 2. We continue the example given in Remark 3 of a Brownian motion fluid flow. Recalling the notation $r:=-U^{+}$and $s:=U^{-}$from [13] and setting $\boldsymbol{r}:=\gamma$ as well as $a:=b$, we obtain

$$
V_{\gamma}(b \mid u)=\frac{\mathrm{e}^{r u}-\mathrm{e}^{s u}}{r \mathrm{e}^{r b}-s \mathrm{e}^{s b}}
$$

which is Equation (2.11) of [13]. Note that, for $\gamma=0$, we obtain

$$
(s, r)= \begin{cases}\left(-2 \frac{\mu}{\sigma^{2}}, 0\right), & \mu>0 \\ \left(0,-2 \frac{\mu}{\sigma^{2}}\right), & \mu<0\end{cases}
$$

This implies that

$$
V_{0}(b \mid u)= \begin{cases}\frac{\sigma^{2}}{2 \mu}\left(\mathrm{e}^{2 \mu b / \sigma^{2}}-\mathrm{e}^{2 \mu(b-u) / \sigma^{2}}\right), & \mu>0, \\ -\frac{\sigma^{2}}{2 \mu}\left(\mathrm{e}^{2 \mu(b-u) / \sigma^{2}}-\mathrm{e}^{2 \mu b / \sigma^{2}}\right), & \mu<0\end{cases}
$$

cf. Equation (2.22) of [13] for the case $\mu>0$.

Example 3. Another example for insurance risk that allows explicit solutions is the compound Poisson model with exponential claim sizes. Interclaim times and claim sizes are assumed to be independent and exponentially distributed with parameters $\lambda>0$ and $\beta>0$, respectively. The rate of premium income is $c>0$. Denote the initial risk reserve by $u \geq 0$. This model has been examined in [12]. Keeping the tilde notation as in Remark 1, the risk reserve at time $t \geq 0$ is given by

$$
\tilde{X}_{t}=u+c t-\sum_{n=1}^{N_{t}} C_{n},
$$

where $\left(N_{t}: t \geq 0\right)$ is a Poisson process with intensity $\lambda$ and the $C_{n}, n \in \mathbb{N}$, are independent and identically distributed (i.i.d.) random variables with exponential distribution of parameter $\beta$. 
We obtain the MMBM $(\mathcal{X}, \mathcal{g})$ as follows. The phase space is partitioned into the subspaces $E_{p}=\{1\}, E_{-}=\{2\}$, and $E_{+}=E_{\sigma}=E_{n}=\varnothing$. The parameters are $\sigma_{1}=\sigma_{2}=0, \mu_{1}=c$, $\mu_{2}=-1$, and

$$
Q=\left(\begin{array}{cc}
-\lambda & \lambda \\
\beta & -\beta
\end{array}\right)
$$

The initial state is $\left(X_{0}, J_{0}\right)=(u, 1)$.

Choose an exit vector $\boldsymbol{r}:=(\gamma, 0)$ with $\gamma>0$, and note that $U^{+}(\boldsymbol{r})$ and $A^{+}(\boldsymbol{r})$ are numbers. The Laplace transform of the first passage time $\tilde{\tau}(x):=\left\{t \geq 0: \tilde{X}_{t}>x\right\}$ to a level $x>u$ is given by $\mathrm{E}\left(\mathrm{e}^{-\gamma \tilde{\tau}(x)} \mid \tilde{X}_{0}=u, \tilde{J}_{0}=1\right)=\mathrm{e}^{U^{+}(\boldsymbol{r})(x-u)}$, where

$$
U^{+}(\boldsymbol{r})=-\frac{\lambda+\gamma}{c}+\frac{\lambda}{c} A^{+}(\boldsymbol{r}) \quad \text { and } \quad A^{+}(\boldsymbol{r})=\frac{\beta}{\beta-U^{+}(\boldsymbol{r})} .
$$

Noting that $U^{+}(\boldsymbol{r})$ must be negative, this resolves as

$$
U^{+}(\boldsymbol{r})=\frac{1}{2 c}\left(c \beta-\gamma-\lambda-\sqrt{\left.(c \beta-\gamma-\lambda)^{2}+4 c \beta \gamma\right)} ;\right.
$$

cf. Equation (3.12) of [12], noting that $\gamma$ is denoted as $\delta$ therein. We further obtain

$$
A^{-}(\boldsymbol{r})=\frac{\beta+U^{-}(\boldsymbol{r})}{\beta} \text { and } U^{-}(\boldsymbol{r})=\frac{1}{2 c}\left(\lambda+\gamma-c \beta-\sqrt{(c \beta-\gamma-\lambda)^{2}+4 c \beta \gamma}\right) .
$$

Hence,

$$
\begin{aligned}
\Psi_{\boldsymbol{r}}^{+}(0, a \mid x) & =\left(\mathrm{e}^{-U^{+}(\boldsymbol{r}) \cdot x}-A^{-}(\boldsymbol{r}) \mathrm{e}^{U^{-}(\boldsymbol{r}) \cdot x} A^{+}(\boldsymbol{r})\right)\left(\mathrm{e}^{-U^{+}(\boldsymbol{r}) \cdot b}-A^{-}(\boldsymbol{r}) \mathrm{e}^{U^{-}(\boldsymbol{r}) \cdot b} A^{+}(\boldsymbol{r})\right)^{-1} \\
& =\left(\mathrm{e}^{\rho x}-\frac{\beta-R}{\beta} \mathrm{e}^{-R x} \frac{\beta}{\beta+\rho}\right)\left(\mathrm{e}^{\rho a}-\frac{\beta-R}{\beta} \mathrm{e}^{-R a} \frac{\beta}{\beta+\rho}\right)^{-1} \\
& =\frac{\mathrm{e}^{\rho x}-\psi(x)}{\mathrm{e}^{\rho a}-\psi(a)},
\end{aligned}
$$

if we write $R:=-U^{-}(\boldsymbol{r}), \rho:=-U^{+}(\boldsymbol{r})$, and $\psi(x):=\mathrm{e}^{-R x}(\beta-R) /(\beta+\rho)$; cf. [12, Equation (6.37)]. This coincides with Formula (6.25) of [12], where $\Psi_{r}^{+}(0, a \mid x)$ is denoted by $B(0, a \mid x)$.

Starting in the ascending phase (collecting premiums), we obtain, for the mean discounted dividends paid before ruin,

$$
\begin{aligned}
V_{\boldsymbol{r}}(a \mid u)= & \left(\mathrm{e}^{-U^{+}(\boldsymbol{r}) u}-A^{-}(\boldsymbol{r}) \mathrm{e}^{U^{-}(\boldsymbol{r}) u} A^{+}(\boldsymbol{r})\right) \\
& \times\left(-U^{+}(\boldsymbol{r}) \mathrm{e}^{-U^{+}(\boldsymbol{r}) a}+A^{-}(\boldsymbol{r}) \mathrm{e}^{U^{-}(\boldsymbol{r}) a}\left(-U^{-}(\boldsymbol{r})\right) A^{+}(\boldsymbol{r})\right)^{-1} \\
= & \left(\mathrm{e}^{\rho u}-\frac{\beta-R}{\beta+\rho} \mathrm{e}^{-R u}\right)\left(\rho \mathrm{e}^{\rho a}+R \frac{\beta-R}{\beta+\rho} \mathrm{e}^{-R a}\right)^{-1} \\
= & \frac{(\beta+\rho) \mathrm{e}^{\rho u}-(\beta-R) \mathrm{e}^{-R u}}{\rho(\beta+\rho) \mathrm{e}^{\rho a}+R(\beta-R) \mathrm{e}^{-R a}},
\end{aligned}
$$

which is Formula (7.8) of [12].

Example 4. With the two exceptions of Examples 1 and 3, there are no explicit closed form solutions to dividend payments available in the literature. We shall, however, shortly sketch 
how to analyse another model of a risk reserve process. Assume that the premium income is given by a Brownian motion $\left(B_{t}: t \geq 0\right)$ with parameters $\mu>0$ for the drift and $\sigma^{2}>0$ for the infinitesimal variance. Claims arrive according to a Poisson process $\left(N_{t}: t \geq 0\right)$ with intensity $\lambda>0$, and the claim sizes $C_{n}, n \in \mathbb{N}$, are i.i.d. with a phase-type distribution of order $m \in \mathbb{N}$ and parameters $(\alpha, T)$. Using the tilde notation of Remark 1, the risk reserve at time $t$ is then given as

$$
\tilde{X}_{t}=u+B_{t}-\sum_{n=1}^{N_{t}} C_{n},
$$

where $u=\tilde{X}_{0}$ denotes the initial risk reserve. Assume that there is a constant dividend barrier at $a>u$, i.e. all the risk reserves exceeding level $a$ will be paid out immediately. Thus, we consider

$$
\tilde{X}_{t}^{a}:=\tilde{X}_{t}-\left(\sup _{s \leq t} \tilde{X}_{s}-a\right)^{+}
$$

for all $t \geq 0$, where $(x)^{+}:=\max (x, 0)$. The time of ruin under this barrier strategy is defined as $\tau_{R}:=\inf \left\{t \geq 0: \tilde{X}_{t}^{a} \leq 0\right\}$. Let

$$
\tilde{S}(t):=\left(\sup _{s \leq t} \tilde{X}_{s}-a\right)^{+}
$$

denote the dividends paid out until time $t$. Then $D:=\tilde{S}\left(\tau_{R}\right)$ is the total amount of dividends paid before ruin.

The MMBM resulting from the fluid embedding mentioned in Remark 1 has phase space $E=E_{\sigma} \cup E_{-}$with $E_{\sigma}=\{1\}$ and $E_{-}=\{2, \ldots, m+1\}$. The parameters are given by $\sigma_{1}=\sigma$ and $\mu_{1}=\mu$, as well as $\sigma_{n}=0$ and $\mu_{n}=-1$ for $n \in E_{-}$. The generator matrix for $\mathcal{g}$ is given by

$$
Q=\left(\begin{array}{cc}
-\lambda & \lambda \alpha \\
\eta & T
\end{array}\right),
$$

where $\eta=-T \mathbf{1}$ and $\mathbf{1}$ denotes the column vector with all entries being 1 . Set the exit vectors $\boldsymbol{r}=\boldsymbol{r}^{\prime}:=(\gamma, 0, \ldots, 0)$. Then the joint distribution of the time until ruin and the dividends paid before ruin is given by

$$
\mathrm{E}\left(\mathrm{e}^{-\gamma \tau_{R}} ; D \in \mathrm{d} x\right)=\Psi_{\boldsymbol{r}}^{+}(0, a \mid u) \mathrm{e}^{G_{r}(a) x} H_{\boldsymbol{r}}(a) \mathrm{d} x
$$

for all $x>0$. We further obtain the singular mass point $\mathrm{E}\left(\mathrm{e}^{-\gamma \tau_{R}} ; D=0\right)=\Psi_{r}^{-}(0, a \mid u)$, caused by the event that ruin will occur before the dividend barrier is hit for the first time.

\section{References}

[1] Asmussen, S. (1995). Stationary distributions for fluid flow models with or without Brownian motion. Commun. Statist. Stoch. Models 11, 21-49.

[2] Asmussen, S. (2003). Applied Probability and Queues (Appl. Math. 51), 2nd edn. Springer, New York.

[3] Asmussen, S. and Kella, O. (2000). A multi-dimensional martingale for Markov additive processes and its applications. Adv. Appl. Prob. 32, 376-393.

[4] Asmussen, S., Avram, F. and Pistorius, M. R. (2004). Russian and American put options under exponential phase-type Lévy models. Stoch. Process. Appl. 109, 79-111.

[5] Asmussen, S., Jobmann, M. and Schwefel, H.-P. (2002). Exact buffer overflow calculations for queues via martingales. Queueing Systems 42, 63-90.

[6] Breuer, L. (2008). First passage times for Markov additive processes with positive jumps of phase type. J. Appl. Prob. 45, 779-799. 
[7] Breuer, L. (2010). A quintuple law for Markov additive processes with phase-type jumps. J. Appl. Prob. 47, 441-458.

[8] Breuer, L. (2010). The total overflow during a busy cycle in a Markov-additive finite buffer system. In Measurement, Modelling, and Evaluation of Computing Systems and Dependability and Fault Tolerance (Lecture Notes Comput. Sci. 5987), eds B. Müller-Clostermann, K. Echtle and E. Rathgeb, Springer, Berlin, pp. 198-211.

[9] Breuer, L. (2012). Occupation times for Markov-modulated Brownian motion. J. Appl. Prob. 49, 549-565.

[10] D'Auria, B., Ivanovs, J., Kella, O. and Mandjes, M. (2012). Two-sided reflection of Markov-modulated Brownian motion. Stoch. Models 28, 316-332.

[11] Frostig, E. (2008). On risk model with dividends payments perturbed by a Brownian motion-an algorithmic approach. ASTIN Bull. 38, 183-206.

[12] Gerber, H. U. ANd Shiu, E. S. W. (1998). On the time value of ruin. N. Amer. Actuarial J. 2, 48-78.

[13] Gerber, H. U. ANd Shiu, E. S. W. (2004). Optimal dividends: analysis with Brownian motion. N. Amer. Actuarial J. 8, 1-20.

[14] Ivanovs, J. (2010). Markov-modulated Brownian motion with two reflecting barriers. J. Appl. Prob. 47, 1034-1047.

[15] Ivanovs, J. (2011). One-sided Markov additive processes and related exit problems. Doctoral Thesis, Universiteit van Amsterdam.

[16] Ivanovs, J. And Palmowski, Z. (2012). Occupation densities in solving exit problems for Markov additive processes and their reflections. Stoch. Process. Appl. 122, 3342-3360.

[17] JiAng, Z. AND Pistorius, M. R. (2008). On perpetual American put valuation and first-passage in a regimeswitching model with jumps. Finance Stoch. 12, 331-355.

[18] LI, S. AND LU, Y. (2007). Moments of the dividend payments and related problems in a Markov-modulated risk model. N. Amer. Actuarial J. 11, 65-76.

[19] Rogers, L. C. G. (1994). Fluid models in queueing theory and Wiener-Hopf factorization of Markov chains. Ann. Appl. Prob. 4, 390-413.

[20] ZHOU, X. (2007). Exit problems for spectrally negative Lévy processes reflected at either the supremum or the infimum. J. Appl. Prob. 44, 1012-1030. 